\title{
JOINT DISORDER: NONREDUCING DISC DISPLACEMENT WITH MOUTH OPENING LIMITATION - REPORT OF A CASE
}

\author{
Hellen Cordeiro CORRÊA ${ }^{1}$, Ana Carolina Stevaneli FrEITAS ${ }^{1}$, Anísio LIMA da SILVA², Túlio Kalife COÊLHO³, \\ Daisilene Baena CASTILLO ${ }^{4}$, Gustavo Helder VINHOLI ${ }^{5}$
}

\author{
1- Graduate Student, Discipline of Prosthodontics, Department of Restorative Dentistry and Prosthodontics, Dental School, Federal University of \\ Mato Grosso do Sul, Campo Grande, MS, Brazil. \\ 2- DDS, MSc, PhD, Full Professor, Discipline of Prosthodontics, Department of Restorative Dentistry and Prosthodontics, Dental School, Federal \\ University of Mato Grosso do Sul, Campo Grande, MS, Brazil. \\ 3- DDS, MSc, Full Professor, Discipline of Prosthodontics, Department of Restorative Dentistry and Prosthodontics, Dental School, Federal University \\ of Mato Grosso do Sul, Campo Grande, MS, Brazil. \\ 4- DDS, MSc, Full Professor, Discipline of Occlusion, Department of Prosthodontics, Dental School, University for the Development of the State and \\ Pantanal Region, Campo Grande, MS, Brazil. \\ 5- DDS, Assistant Professor, Discipline of Occlusion, Department of Restorative Dentistry and Prosthodontics, Dental School, Federal University of \\ Mato Grosso do Sul, Campo Grande, MS, Brazil.
}

Corresponding address: Gustavo Helder Vinholi - Departamento de Odontologia Restauradora e Prótese - Faculdade de Odontologia "Albino Coimbra Filho", UFMS - Av. Senador Filinto Müller, s/nº - Bairro Vila Ipiranga - Caixa Postal 549 - 79080-190 - Campo Grande, MS, Brasil - Phone/Fax: +5567-3345-7681 - e-mail: gvinholi@yahoo.com.br

Received: June 4, 2008 - Modification: August 30, 2008 - Accepted: October 7, 2008

\begin{abstract}
$T$

he internal derangement of the temporomandibular joint (TMJ) represents $8 \%$ of all cases of temporomandibular disorders (TMD) posing difficulties to establish an accurate diagnosis and treatment because of its low prevalence. This article presents the case of an 18-year-old Caucasian female patient who came to our Orofacial Pain and TMD Outpatient Service with complaints of intense pain on the right TMJ and limitation of mouth opening (maximum interincisal opening of $29 \mathrm{~mm}$ ) with deviation to right, which she had been experiencing for the past 3 years. After a detailed clinical interview, a diagnosis hypothesis of nonreducing disc displacement with mouth opening limitation was established. The proposed treatment consisted of intra-joint infiltration with anesthetic in the right TMJ followed by jaw manipulation to recapture the articular disc, which was impeding the complete translation movement of the affected TMJ. After jaw manipulation, a new evaluation was done and showed the re-establishment of jaw dynamics with mouth opening and closing without deviation to the right side, clicking, opening limitation or pain. The patient was followed up at 6 months intervals. Two years after treatment, the patient was reevaluated and her mandibular range of motion without aid increased to $54 \mathrm{~mm}$ with no clicking, deviation to right, trismus or pain on the TMJ, indicating success of the treatment approach without recurrence of the pathology.
\end{abstract}

Key words: Temporomandibular. Disc displacement. Mouth opening imitation.

\section{INTRODUCTION}

Temporomandibular disorders (TMD) is a collective term that includes a large spectrum of clinical problems of the joint and the muscles on the orofacial area; these dysfunctions are characterized mainly by pain, sounds in the temporomandibular joint (TMJ) and irregular or limited function of the jaw. It is comprised in a distinct subgroup of musculoskeletal and rheumatology disorders, representing an important cause of non-dental pain on the orofacial area ${ }^{2,4}$.

TMJ dysfunctions play an important role in the production of TMD signs and symptoms and have been extensively discussed on the literature. The problems in TMJ were the initial focus of the development of the concepts of TMD. A study by Costen ${ }^{3}$ (1997) was the initial mark of this research line, since this author listed a number of symptoms associated with the distal displacement of the jaw and attributed this etiology to a great reduction of the mouth opening after loss of the teeth. Much earlier, in the 50s, the interest was directed to the masticatory muscles, which were considered to be the most frequent source of TMD signs and symptoms ${ }^{7}$. Later in the 80 s, several authors reported that the joint disorders were the most prevalent factors in TMD, however, currently, many authors affirm that a variety of dysfunctions can cause pathologies in the TMJ and masticatory muscles acting separately or together ${ }^{2}$. 
A previous study ${ }^{1}$ that made a characterization of patients in a TMD and orofacial pain outpatient clinic found that $8 \%$ of the diagnosed cases involved only the TMJ, $73 \%$ involved only the masticatory muscles and $19 \%$ had both types of disturbances. According to this study, about 8 million people in Brazil have some degree of TMD, $90 \%$ of this total are women aged 20 to 45 years. According to Dworkin and LeResche ${ }^{5}$ (1992), TMD are classified in three types: 1. Masticatory Muscle Dysfunction: muscular disorders and muscular disorders with mouth opening limitation; 2. Internal TMJ Derangement: disc displacement with reduction, nonreducing disc displacement with mouth opening limitation; nonreducing disc displacement without mouth opening limitation; and 3. Degenerative diseases: osteoarthritis and osteoarthrosis.

By scientific definition, nonreducing disc displacement with mouth opening limitation is a condition in which the original disc is moved from its position between the condyle and the articular fossa to a forward, medial or lateral position, associated with limitation of mouth opening ${ }^{2,4,5}$. According to several authors ${ }^{2,4,5,11}$, this pathology presents with pathognomonic signs and symptoms: History of significant mouth opening limitation; Maximum mouth opening without aid of $35 \mathrm{~mm}$ or less; Maximum mouth opening with aid of $4 \mathrm{~mm}$ or less in relation to opening without aid; Limited opening with shift without correction of the jaw to the side of the affected TMJ; Absence of joint sounds (the patient usually reports history of reciprocal click in the affected TMJ, which was interrupted after jaw locking; Pain on the pre-auricular region during jaw function and mastication of hard foods; Pain on palpation and during functional joint tests on the affected TMJ. Still according to the abovementioned authors ${ }^{2,4,5,11}$, if all these pathognomonic signs and symptoms are present, complementary imaging exams are not necessary to establish a diagnosis. This article presents the case of a young patient who sought treatment at our Orofacial Pain and TMD Outpatient Service due to intense pain on the right TMJ and mouth opening limitation for the past 3 years, and was diagnosed as having nonreducing disc displacement with mouth opening limitation based on the clinical signs and symptoms.

\section{MATERIAL AND METHODS}

An 18-year-old Caucasian female patient came to the Orofacial Pain and TMD Outpatient Service of the Dental School of the Federal University of Mato Grosso do Sul, Brazil with complaints of extreme pain on the right preauricular region, limitation of mouth opening (maximum interincisal opening of $29 \mathrm{~mm}$ ) and jaw movement with deviation to the right side (Figure 1). The pain had begun strong and intense and the last pain episode occurred 45 days before the consultation. The patient reported having suffered from these conditions for the past 3 years. A pulsating and twinge pain was described on the right ear with a frequency of 7 days a week. It used to begin in the morning and lasted the whole day until the end of the night.
The patient informed that the pain intensity increased when she chewed hard foods or did any mandibular function or parafunction. Not speaking and not doing any jaw function alleviated the pain, while stress, chewing hard foods and parafunction worsened the painful sensation.

After a detailed clinical interview, a diagnosis hypothesis of nonreducing disc displacement with mouth opening limitation $^{5}$ was established. The proposed treatment consisted of intra-joint infiltration with anesthetic in the right TMJ followed by jaw manipulation to recapture the articular disc, which was impeding the complete translation movement of the affected $\mathrm{TMJ}^{2}$. The patient was infiltrated with $3 \%$ prilocaine with felipressin $\left(0.03 \mathrm{IU} / \mathrm{mL}\right.$; Citanest ${ }^{\circledR}$; Astra Química do Brasil Ltda., São Paulo, SP, Brazil) in a $1.8 \mathrm{~mL}$ cartridge packed in a Carpule syringe (Duflex, S.S.White, Rio de Janeiro, RJ) with a long needle (Figure 2). The applied technique consisted of a modification of the arthrocentesis technique proposed by Nitzan, et al. ${ }^{9}$ (1991), in which infiltration of the anesthetic solution was done without the presence of the aspiration clyster - pipe. The tragus line was delimited by the edge of the eye and the first

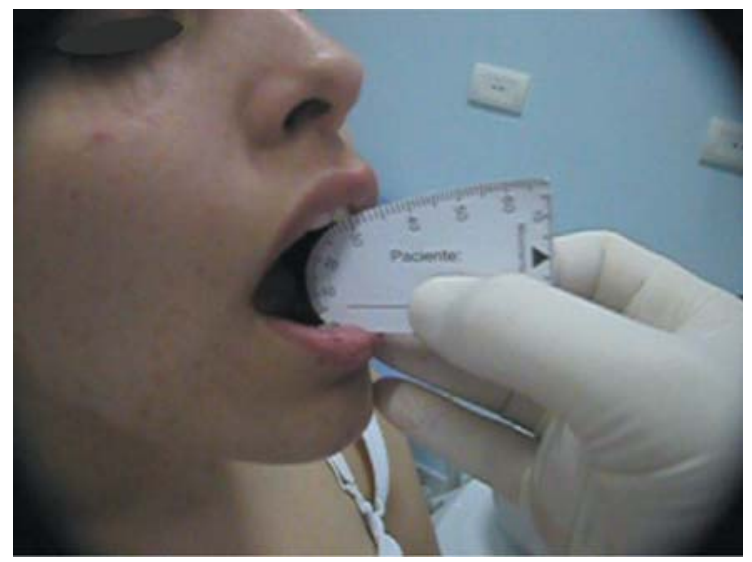

FIGURE 1- limitation of mouth opening and jaw movement with deviation to the right side

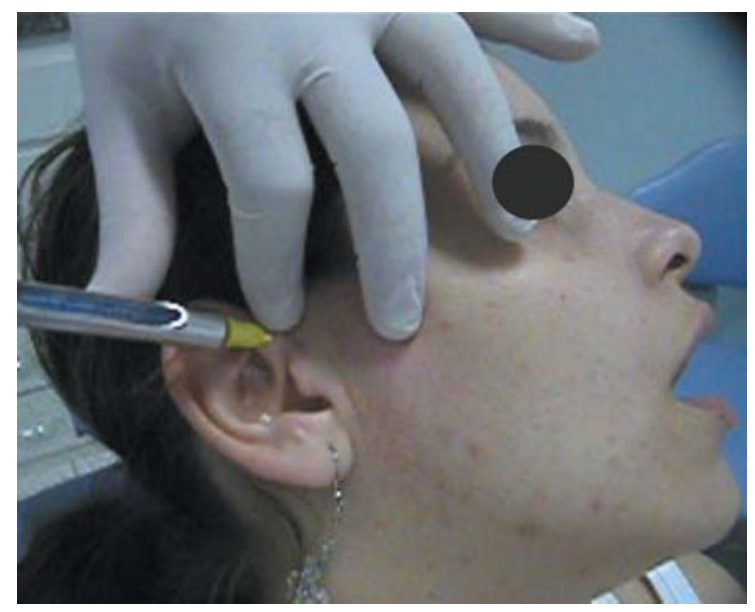

FIGURE 2- Infiltration of the anesthetic solution 


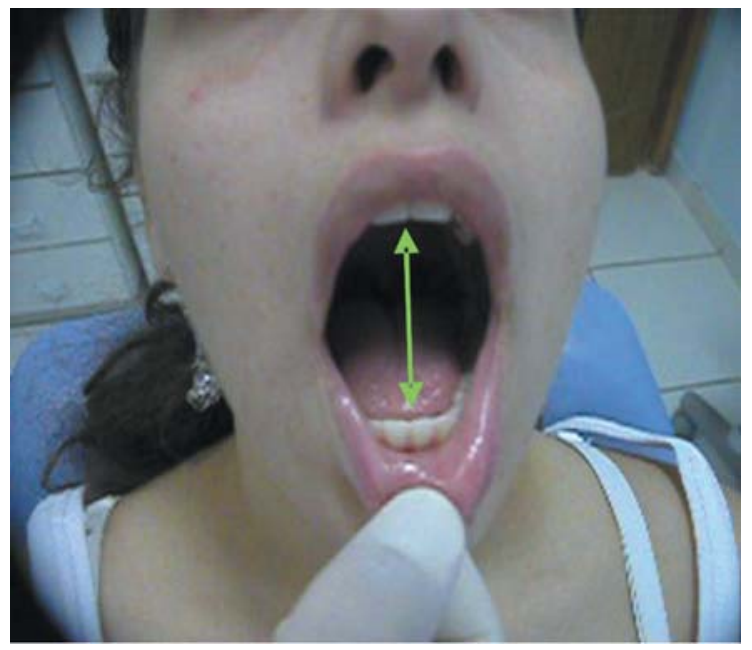

FIGURE 3- Re-establishment of the normal jaw dynamics

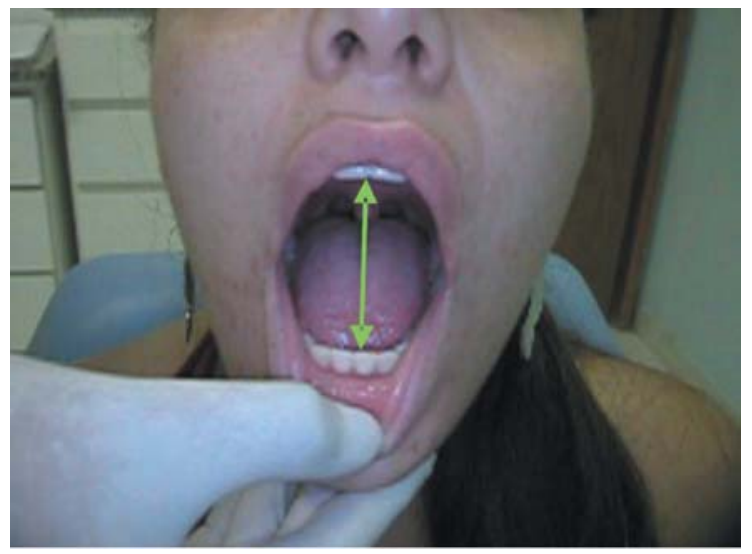

FIGURE 4- Mandibular range of motion two years after treatment

demarcation was done $10 \mathrm{~mm}$ distant from the tragus and $0.5 \mathrm{~mm}$ below the drawn line. The patient was kept with the mouth opened during the whole procedure in such a way the anesthetic solution could be introduced into the upper compartment of the joint cavity, which remained empty, and then the condyle was positioned forward. After the anesthetic procedure, the jaw was manipulated to recapture the articular disc that was in an anterior position and without reduction. A passive manipulation was performed with anti-clockwise movements. A strong clicking sound was heard during the process, indicating successful disc recapture.

After jaw manipulation, new evaluation showed the reestablishment of jaw dynamics with mouth opening and closing without deviation, clicking, trismus or pain (Figure 3). The mandibular range of motion without aid was $51 \mathrm{~mm}$ with no pain on the right TMJ. As a complement of the therapy, a front-plateau was fabricated in the same session in order to maintain the jaw totally onward during 2 days of continuous use and recover the disc's original anatomic "bow tie" shape. A non-hormonal antiinflammatory drug of selective inhibition type (Arcoxia ${ }^{\circledR} ; 90$ mg; Merck Sharp \& Dohme, São Paulo, SP, Brazil) was prescribed once a day during 7 days associated with cryotherapy on the right TMJ for $10 \mathrm{~min}, 3$ times a day during 7 days.

The patient was followed up at 6 months intervals. Two years after treatment, the patient was reevaluated and her mandibular range of motion without aid increased to $54 \mathrm{~mm}$ with no clicking, deviation to right, trismus or pain on the TMJ, indicating success of the treatment approach without recurrence of the pathology (Figure 4).

\section{DISCUSSION}

This treatment protocol for internal TMJ derangement is a new modality of conservative surgery. In the past, several cases of disc displacements and jaw trismus which had been treated conservatively using interocclusal splints, thermotherapy, physical therapy, cryotherapy and drugs, and that had not reached the desired outcome, were referred to surgery and subjected to more aggressive procedures, such as disc anchorage and/or TMJ arthroplasty ${ }^{15}$.

The therapeutic approach used in the present case emerges as an intermediate alternative between conservative and surgical treatment. The advantages of this therapy are conveyed in the simplicity of the technique applied, and also, in the fact of being completely reversible, of lower cost and performable in the dental clinic. Washing the upper joint space reduces the pain due to removal of the inflammatory mediators acting in the joint ${ }^{12}$. There is an increase on jaw mobility for the rupture of the intra-joint adhesions $^{14}$ and the improvement of disc mobility, which reduces the mechanical obstruction caused by the anterior disc position ${ }^{8}$. Jaw manipulation aims at recapturing the disc or at least breaking its adhesions, which is expected to allow for the return of lubrication by the sinovial liquid and resorption of the persistent inflammatory fluid ${ }^{2,11}$.

As a complementation of the treatment, the front-plateau blocks the jaw in an advanced position allowing the capsular ligament to come to an stabilization and as consequence provide the remodeling of the bilaminar area and permit that the disc recovers its original anatomic "bow tie" shape, by the prolongation of the previous recess and compression of its central portion ${ }^{6,10,11,13}$. In the case presented in this paper, the treatment approach was successful with improvement of the symptomatology associated with internal TMJ derangement and increase of the mandibular range of motion.

\section{CONCLUSION}

It was possible to observe an effective improvement and recovery of the patient who underwent the proposed therapeutic protocol. 


\section{REFERENCES}

1- Bove SR, Guimarães AS, Smith RL. Characterization of patients in a temporomandibular dysfunction and orofacial pain outpatient clinic. Rev Lat Am Enfermagem. 2005;13(5):686-91.

2- Carlsson GE, Magnusson T, Guimarães AS. Management of temporomandibular disorders in the general dental practice. Chicago: Quintessence; 2006.

3- Costen JB. A syndrome of ear and sinus symptoms dependent upon disturbed function of the temporomandibular joint. Ann Otol Rhinol Laryngol. 1997;106(10 pt 1):805-19.

4- DeBoever JA, Carlsson GE. Etiology and differential diagnosis. In: Zarb GA, Carlsson GE, Sessle BJ, Mohl ND, editors. Temporomandibular joint and masticatory muscle disorders. 2nd ed. Copenhagen: Munksgaard; 1994.

5- Dworkin SF, LeResche L. Research diagnostic criteria for temporomandibular disorders: review, criteria, examinations and specifications, critique. J Craniomandib Disord. 1992;6(4):301-55.

6- Farrar WB, McCarty WL. A clinical outline of temporomandibular joint diagnosis and treatment. Montgomery: Normandie Publications; 1982.

7- Linton SI, Hellsing Al, Andersson D. A controlled study of the effect of an early intervention on acute musculoskeletal pain problems. Pain. 1993;54(1):353-9.

8- Moses JJ, Sartoris D, Glass R, Tanaka T, Poker I. The effect of arthroscopy surgical lysis and lavage of the superior joint space on temporomandibular joint disc position and mobility. J Oral Maxillofac Surg. 1989;47(7):674-8.

9- Nitzan DW, Dolwick MF, Martinez GA. Temporomandibular joint arthrocentesis: a simplified treatment for severe, limited mouth opening. J Oral Maxillofac Surg. 1991;49:1163-7.

10- Okeson JP. Management of temporomandibular disorders and occlusion. 6th ed. Saint Louis: Mosby-Elsevier; 2008.

11- Palla S. Mioartropatias del sistema mastigatório e dolores orofaciais. São Paulo: Artes médicas; 2004.

12- Quinn JH, Bazan NG. Identification of prostaglandin E2 and leukotriene B4 in the synovial fluid of painful, dysfunctional temporomandibular joints. J Oral Maxillofac Surg. 1990;48(9):968-71.

13- Shoji YN. Nonsurgical treatment of anterior disc displacement without reduction of the temporomandibular joint: a case report on the relationship between condylar rotation and translation. Cranio. 1995;13(4):270-3.

14- Spallaccia F, Rivaroli A, Cascone P. Temporomandibular joint arthrocentesis: long-term results. Bull Group Int Rech Sci Stomatol Odontol. 2000;42(1):31-7.

15- Vasconcelos BEV, Bessa-Nogueira RV, Rocha NS. Artrocentesis of temporomandibular joint: evaluation of the results and review of literature. Rev Bras Otorrinolaringol. 2006;72(5):634-8. 